\title{
Neuropsychiatric symptoms in Parkinson's disease
}

\author{
D. Aarsland ${ }^{1,2,3}$, L. Marsh ${ }^{4,5,6}$, and A. Schrag ${ }^{7}$
}

${ }^{1}$ The Norwegian Centre for Movement Disorders, Stavanger University Hospital, Stavanger, Norway ${ }^{2}$ Department of Clinical Medicine, University of Bergen, Bergen, Norway ${ }^{3}$ Institute of Psychiatry, King's College London, London, England ${ }^{4}$ Department of Psychiatry and Behavioral Sciences, Johns Hopkins University School of Medicine, Baltimore, Maryland, USA ${ }^{5}$ Department of Neurology and Neurological Sciences, Johns Hopkins University School of Medicine, Baltimore, Maryland, USA ${ }^{6}$ Morris K. Udall Parkinson's Disease Research Center of Excellence at Johns Hopkins, Johns Hopkins University School of Medicine, Baltimore, Maryland, USA ${ }^{7}$ Department of Clinical Neurosciences, University College, London, England

\section{Abstract}

Neuropsychiatric symptoms are common in Parkinson's disease, even at the earliest stages, and have important consequences for quality of life and daily functioning, are associated with increased carer burden and increased risk for nursing home admission. In addition to cognitive impairment, a wide range of neuropsychiatric symptoms have been reported. In this paper, the epidemiology, clinical course, diagnosis, and management of some of the most common neuropsychiatric symptoms in PD are discussed: depression, anxiety, apathy, fatigue and psychotic symptoms. Although much is known regarding the prevalence and course of these symptoms, the empirical evidence for how to manage these symptoms is limited at best. There is thus an urgent need for systematic studies for the pharmacological and non-pharmacological management of these symptoms.

\section{Introduction}

Neuropsychiatric symptoms including depression, anxiety, apathy and psychosis[1] are common in Parkinson's disease (PD), affecting the majority of patients at some time during the course of disease. Among the most common, and most important, neuropsychiatric symptoms in PD are depression, anxiety, apathy and psychosis[1].In this paper which was solicited from the Movement Disorders Society Meeting in Chicago in 2008, we will review the epidemiology, clinical characteristics, and management of these neuropsychiatric symptoms in PD.

\footnotetext{
Correspondence to: Prof. Dag Aarsland, The Norwegian Centre for Movement Disorders, Stavanger University Hospital, PO Box 8100, N-4068 Stavanger, Norway. daarsland@ gmail.com. Tel. 0047-51515062. Fax 0047-51515515..

Author roles Research project: This was an invited review based on the syllabi of a session on "Neuropsychiatric symptoms in Parkinson's disease -Beyond Dementia" at the Movement Disorders conference in Istanbul, 2008.

Statistical Analysis: Not applicable.

Manuscript: Anette Schrag wrote the section on Depression and Anxiety, Laura Marsh the Section on Fatigue and Apathy, and Dag Aarsland the section on Psychosis. All authors reviewed and approved the final manuscript.

No conflicts of interest to report
} 


\section{Depression and Anxiety}

\section{Prevalence and pattern of occurrence}

Symptoms of depression and anxiety are common in Parkinson's disease (PD), and depression scores are closely associated with patient-perceived health-status in patients with PD[2]. Approximately 30-40\% of patients with PD have significant depressive symptoms, with lower prevalence rates in population-based studies[3-5]. Anxiety affects up to $40 \%$ of patients with PD[6-11]. Symptoms of both depression and anxiety can be off-period related phenomena, which are usually, but not always, seen in parallel to motor fluctuations, and can then be particularly severe but respond to antiparkinsonian medication[12-15].

Both depression and anxiety can predate motor symptoms by several years and belong to the non-motor features that may herald the development of PD[16]. Whilst depression may occur reactively, or coincidentally, there is no doubt that at least in part it is an integral part of PD. The pathophysiology of these complications is complex and probably includes dopaminergic, serotonergic and noradrenergic mechanisms. Postmortem studies have implicated serotoneric and noradrenergic structures such as the raphe nuclei and locus coeruleus, which are thought to be involved in the development of depression and are both involved early in the course of PD $[17,18]$. Cerbrospinal fluid levels of the serotonin metabolite 5-hydroxyindoleacetic acid have also been correlated with severity of depression in $\mathrm{PD}$ [19]. There is considerable evidence that dopaminergic dysfunction also plays a role. Dopaminergic projections from the ventral tegmentum of the midbrain to the medial temporal and orbitofrontal regions have been shown to be affected in post mortem studies[20]. In addition, there is evidence from imaging studies that limbic noradrenergic/ dopaminergic pathways are dysfunctional in PD patients with depression compared to those without[21]. There also appears to be a genetic predisposition, as first degree relatives of patients with PD are more likely to have had depression or anxiety[22].

\section{Clinical characteristics of depression and anxiety in PD}

The key characteristics of depression are low mood and lack of interest or pleasure (anhedonia), one of which is required for a diagnosis of depression in most classifications. Other features of depression can be present in varying combinations but many of these, such as altered appetite or sleep, weight change, loss of libido, psychomotor retardation, reduced memory and loss of energy can overlap with the symptoms of PD, making diagnosis of depression in PD difficult. Some features of depression may also be less common in patients with PD, such as feelings of guilt or worthlessness and suicidal ideation [23,24]. However, in patients who have undergone subthalamic nucleus (STN) deep brain stimulation (DBS), suicide rate is increased, and was the most common cause of post-operative mortality in a study of more than 5,000 patients who underwent this procedure[25], and vigilance towards occurrence of suicidal ideation has been advised[26-29].

Particularly in patients with advanced PD, there can be considerable difficulty in distinguishing somatic symptoms of depression from those of PD, such as slowness of movement and thinking, loss of appetite and weight, or sleep problems. The association with other symptoms of depression or PD and specific symptom characteristics (e.g. fatiguability of movement in bradykinesia vs overall slowness in psychomotor retardation, the pattern of slowness of thinking in depression as opposed to that in cognitive impairment, or the temporal pattern of sleep disturbances and their association with immobility) can be helpful in distinguishing whether symptom are due to PD or depression. However, this differentiation can be difficult even for an experienced clinician.

The most common anxiety disorders in PD are panic attacks (often during off-periods), generalised anxiety disorder (GAD), simple and social phobias. Outside off-periods, there is 
no clear relationship to severity of motor symptoms or dementia, but anxiety may be part of an underlying depressive disorder.[30] The relationship of antiparkinsonian medication to anxiety in PD needs further clarification.

\section{Diagnosis, Assessment and Classification}

The most commonly used diagnostic classification for depression and anxiety is the DSM IV-TR[31]. As application of these criteria may miss a substantial number of patients with depression in PD (dPD), an NINDS/NIMH working group on depression and PD[32] issued the following recommendations: I) The diagnosis of dPD should be made according to DSM diagnostic criteria using an inclusive approach, using all symptoms irrespective of potential aetiological basis, as it has the greatest sensitivity and reliability and does not require clinical judgment; II) subsyndromal depression should be included as a diagnostic category in research studies; III) the timing of assessment should be specified ("on" versus "off"periods) (it should however be noted that most instruments specify $\geq 2$ weeks as a time frame); IV) informants should be used for cognitively impaired patients; and V) anhedonia should only be diagnosed based on loss of pleasure rather than loss of interest (as it overlaps with apathy) for diagnosis of minor depression/subsyndromal depression. These modified DSM criteria require further validation. As there is significant association between low mood and anxiety symptoms, it has also been suggested by others that these should be added to the DSM diagnostic framework[33]. The diagnosis of minor depression is based on the same symptoms, but only requires presence of 2-5 symptoms in the DSM IV-TR classification, and dysthymia requires that depressed mood has been present on most days for at least two years with at least two out of 6 other symptoms.

Clinimetric rating scales are often used in large-scale epidemiological and other studies as they do not require clinical judgment and are quantifiable. As they have diverse properties and differing clinical applicability their use should be tailored to the purpose of the individual study or purpose (see table 1). Generally, observer-rated scales are preferred if the study or clinical situation permits. For screening purposes, the Hamilton Depression Scale (HAM-D), the Beck Depression Inventory (BDI), the Hospital Anxiety and Depression Scale (HADS), the Montgomery-Asberg Depression Rating Scale (MADRS) and Geriatric Depression Scale (GDS) have been shown to be valid in depression in PD. For measurement of severity of depressive symptoms, the Ham-D, MADRS, BDI and Zung Self-Rating Depression Scale (SDS) scales are useful instruments. The Cornell Scale for the Assessment of Depression in Dementia (CSDD) is valid for the assessment of depression in patients with dementia. However, a diagnosis of depression should only be made using clinical criteria rather than scales. As the differentiation of overlapping symptoms of depression and PD remains difficult, clinical ratings scales should be used only with adjustment for severity and change in PD symptoms using appropriate scales (e.g. UPDRS).[34]

The application and clinical validity of DSM anxiety criteria in PD has not undergone the same systematic analysis and critique as depression but suffers the same conceptual difficulties and problems with symptomatic overlap. In addition, it should be noted that social phobia should not be diagnosed if the fear is only about being embarrassed by PD symptoms in public. Several rating scales for anxiety have been used in patients with PD, but their validity needs to be assessed further[8].

\section{Management}

The first important issue is to determine whether the depression and/or anxiety symptoms occur solely in off-periods (see above). If so, adjustment of antiparkinsonian medication is required and usually successful (personal observation). This may be obvious, but not 
uncommonly prolonged neuropsychiatric symptoms are due to under-medication and may be difficult to identify as off-period related.

If depression or anxiety symptoms are not due to non-motor fluctuations, the severity of symptoms should be determined to assess the need for treatment. In most cases with mild depression (which are the majority), non-pharmacological intervention is the treatment of choice, with treatments ranging from counselling and patient education to cognitivebehavioural therapy[35].

One should bear in mind when assessing open-label studies and individual patient response, that there is a very large placebo-response in response to antidepressant trials in PD. There is a lack of large, well-designed randomised placebo-controlled trials of antidepressant and anxiolytic treatments[36-38]. There are several approaches to treatment of dPD: psychotropic medication, dopaminergic medication, electroconvulsive therapy (ECT) and repetitive transcranial magnetic stimulation (rTMS).

Dopaminergic_-As many patients will already be on dopaminergic medication and/or also require improvement of motor symptoms, optimisation of existing treatment may be the most pragmatic first step. Although no class I evidence from controlled trials in PD is available at the time of writing, dopaminergic medications have been suggested to improve depressive symptoms in PD. Pramipexole has been reported in double-blind trials to be effective for both unipolar and bipolar depression in non-PD patients. In PD, several studies without placebo control reported improvement of depression scores in depressed patients with PD, and a double-blind trial of ropinirole extended-release preparation reported improvements in depression scores[39]. However, in the double-blind ropinirole study the patients were not selected according to criteria for depression and the primary study outcome was motor function. In addition, given the overlap of features of depression and $\mathrm{PD}$, the antidepressant effect of these medications is difficult to ascertain since the studies did not control for improvement of motor symptoms. The results of an ongoing placebocontrolled trial of pramipexole in depression in PD are currently awaited.

Psychotropic-Pharmacological agents used for dPD include tricyclic antidepressants, tricyclic-related drugs (trazodone), selective serotonin reuptake inhibitors (SSRI), the serotonin and noradrenaline re-uptake inhibitor (SNRI) venlafaxine, the selective noradrenaline re-uptake inhibitor reboxetine, and the pre-synaptic alpha-2 adrenoreceptor antagonist mirtazapine. There is a suggestion that antidepressant medications in PD are less effective than in depressed non-PD patients, although older patients and those with major depression may have a better response[38]. In one meta-analysis (11 studies, 2 placebocontrolled)[38], there was a large composite reduction in depression rating scales in both active treatment and placebo groups but no statistically significant difference between the two groups. In another (only 6 six studies met criteria for inclusion, 3 placebo-controlled) [40], it was concluded that there is some evidence for the antidepressant effectiveness of amitriptyline in PD patients (with the caveat of potential side effects) and that SSRI have not been demonstrated to have clear efficacy compared to placebo. In clinical practice nortriptyline is generally preferred in practice to amitryptiline due to its better side effect profile. A small recently published placebo-controlled study reported that nortriptyline was more effective than placebo in improving depressive symptoms, but there was no significant benefit in the group of patients taking paroxetine CR compared to the placebo group[41]. On the other hand, another small placebo-controlled trial found that both desipramine, a predominantly noradrenergic reuptake inhibitor tricyclic, and citalopram led to improvement of depressive symptoms after 30 days[42]. 
Consideration should be given to the potential side effects related to these medications, particularly in the elderly, their possible interaction with other drugs (particularly those metabolised via the cytochrome $\mathrm{P} 450$ system) resulting in variations in plasma concentrations, reports of exacerbation of motor function and their potential to exacerbate non-motor and dopaminergic drug-related complications of the disease. Tricyclics are generally less well tolerated than SSRIs[42]. SSRIs potentially have important pharmacological interactions with the MAO-B inhibitors selegiline and rasagiline and thus theoretical predilection to a serotonin-syndrome. This effect is likely to be rare given that at therapeutic doses MAO-B inhibitors produce no significant MAO-A inhibition, but should be considered.

Atypical neuroleptics may have a role in treating depression with co-morbid psychotic features, although these may respond to antidepressants alone[43].

There has been no systematic assessment of anxiolytic treatment in PD. The SSRI are currently favoured as treatment of anxiety disorders in PD but their therapeutic efficacy is yet to be demonstrated. Benzodiazepines should be used with caution as they can increase the risk of falls and cognitive, autonomic and sleep-related side effects are likely to be common in PD (although clonazepam can be useful as a treatment of REM-sleep behaviour disorder), and clomipramine shares the anticholinergic side-effect of other tricyclics. Small studies have reported effects of neuromuscular (massage) therapy, STN-DBS and psychotherapeutic treatment $[8,44,45]$. Anxiolytic treatments that have not yet been assessed in PD include pregablin and venlafaxine.

\section{Other options}

A small placebo-controlled pilot trial recently reported significantly greater improvement of depression in patients treated with omega-3 fatty-acid supplementation[46]. Bright light therapy has also been reported to be slightly superior to placebo (with less bright light) in a trial[47]. The results of these small trials require further study.

STN-DBS has been reported to improve depression and anxiety scores in the majority of patients who underwent this procedure for improvement of motor symptoms, but significant psychiatric disease has been an exclusion criterion in most studies, and suicidal ideation is a risk following this procedure (see above). At present, this should therefore not be considered a treatment option for depression or anxiety in PD.

rTMS has been reported as a promising option for $\mathrm{dPD}$, but no large controlled trials are currently available[48-50].

ECT is reserved for severe, treatment resistant cases of depression, and can be a life-saving procedure in selected cases[51-53]. However, no sufficient trials in PD are available.

\section{Apathy and fatigue}

\section{Introduction}

Apathy and fatigue are two common non-motor disturbances in patients with Parkinson's disease (PD) that contribute substantially to disability and are attributed to basal ganglia pathology and disturbances in frontal-subcortical connections[54]. For both, mood symptoms and cognitive deficits are also frequently present. Appreciation of the overlap among motor, cognitive, and psychiatric phenomena along with the specific features of apathy and fatigue in patients with PD facilitates recognition of each and the likelihood that symptoms will be addressed. However, only a few studies have addressed apathy or fatigue in PD and improved detection and effective treatments are needed. 


\section{Prevalence and pattern of occurrence}

Apathy and fatigue can present as a feature of another condition or as independent and clinically significant symptoms. Most studies have used symptom rating scales to define the presence of apathy. Reported prevalence, ranging from $17 \%$ to $70 \%[1,55-59]$, is influenced by the extent of cognitive impairment and depressive symptoms in the sample, the assessment tools used, and the rater (i.e., a clinician, family member, or patient) $[1,57,59,60]$. Apathy symptoms in the absence of depression occur in $4 \%$ to $30 \%$ (14\% average prevalence), whereas reported prevalence for depression in the absence of apathy is $6 \%$ to $28 \%$ and for the combination of apathy and depression is $12 \%$ to $47 \%[1,54-58]$. Apathy, also a feature of dementia, delirium, and demoralization, and can occur in the absence of cognitive decline[4,54]. The longitudinal course of apathy has not been studied.

Fatigue, the single most disabling symptom reported by up to $1 / 3$ of patients with $\mathrm{PD}[61,62]$, has a prevalence of $32 \%$ to $58 \%$ that is also influenced by inconsistent definitions of fatigue and which assessment instrument was used[61,63,64]. Fatigue is present early in the course of PD and may even predate onset of motor features[65]. Once present, fatigue can be chronic or intermittent, but lifetime prevalence increases over time[64]. Fatigue in PD is also associated frequently with depression, cognitive deficits, and daytime sleepiness[64,66,67]. Despite its prevalence and impact, fatigue is under-recognized clinically[68].

\section{Clinical characteristics}

Apathy refers to a set of behavioral, emotional and cognitive features that involve reduced interest and motivation in goal-directed behaviors, indifference, and flattened affect[69]. Some studies emphasize lack of motivation whereas others focus on lack of emotional responsiveness as a core feature. Patients typically show poor motivation with reduced initiative, effort, and perseverance as well as indifference to their circumstances. This is manifest as a lack of spontaneous engagement or early withdrawal in activities, a lack of concern for ones own health, or the absence of curiousness about others or new experiences. The impact of apathy is considerable; the patient is generally inactive, and this passivity leads to further functional decline and greater debility $[1,55,58,59]$. Families misattribute signs of apathy as evidence of laziness, entitlement, or contrariness. Thus, providing care can be unrewarding and lead to resentment, especially when apathy remains undiagnosed[60].

The role of depressive disturbances in the presentation of apathy is an important consideration. Reports are inconsistent as to whether the combination of apathy and depression is more common than apathy without depression or depression without apathy[54,57,58]. At issue is whether loss of goal-oriented cognition, a feature of Marin's criteria for an apathy diagnosis[70], qualitatively represents the same phenomenon as the reduced interest and anhedonia that is characteristic of depressive disorders. In addition to overlapping diagnostic criteria, symptom rating scales also include items that overlap features of depression and apathy. The Neuropsychiatric Inventory is an exception in this regard, but apathy and depressive subscores were still significantly correlated in a study involving PD subjects[55]. By contrast, apathy and depression were more clearly dissociated in other disorders in that study, such as progressive supranuclear palsy.

Cognitive impairment, another important clinical factor in apathy, is also a general feature of PD. Apathy in PD is associated with bradyphrenia, global cognitive impairment, and executive dysfunction[54,56,59]. However, motor severity, 1-dopa dose, physical disability, and PD duration are less likely to be associated with apathy[56,58]. 
There are two main classifications of fatigue: peripheral and central[43]. Peripheral fatigue is a physiological phenomenon that involves lack of energy associated with muscular fatigue. It is objectively measured by decreased force generation or the inability to sustain repetitive movements[65]. More clinically relevant in most cases is central fatigue, which is generally described as an abnormal degree of persistent tiredness, weakness, or exhaustion that is mental, physical or both in the absence of motor or physical impairment.[65] Central fatigue is a subjective experience (in contrast to apathy, which is generally an observed phenomenon) with two subtypes, physical and mental. Physical fatigue represents the sense of physical exhaustion and lack of energy to perform physical tasks despite ability to do so. Mental fatigue refers to the effects experienced during and after prolonged periods of demanding cognitive activities that require sustained mental efficiency. Given its subjective nature, the overlap between physical and mental fatigue is not always clear.

In interviews of patients with PD, fatigue was described as unpredictable with respect to its onset, duration, and relationship to prior activity, but often exacerbated by physical, psychological, or social stressors[71]. Inability to initiate and sustain activity associated with fatigue is distinct from sadness, sleepiness, or impaired motor function[71]. Fatigue has adverse effects on quality of life, depression, and disability in PD[72] and is the primary determinant of work-related disability[73]. Relationships between fatigue and motor symptoms, daytime sleepiness, sleep quality, and physical activity are inconsistent[60,64]. Surprisingly, fatigue is unrelated to exercise efficiency, activity level, or physical fatigue[65].

Fatigue in patients with PD is associated with higher rates of depressive symptoms, sleep disturbances, and cognitive disturbances, but it is also highly prevalent in non-depressed patients[64]. In one series, $43.5 \%$ of patients without depression, dementia, or sleep problems still reported fatigue, in contrast to $4.5 \%$ of controls[74]. By definition, mental fatigue involves difficulties initiating and maintaining sustained cognitive performance and is often associated with motivational and attentional difficulties[65]. For example, mental fatigue during driving may lead to reduced error monitoring In contrast to apathy, which appears to be associated with pre-existent cognitive impairment, especially executive dysfunction, fatigue occurs as a consequence of mental challenges. In particular, tasks involving increased attentional demands and multi-tasking may be more likely to be associated with fatigue[67].

\section{Diagnosis, Assessment and Classification}

For both apathy and fatigue, co-existence of mood symptoms and cognitive deficits, and their overlap with motor signs of PD, contribute to diagnostic challenges. For example, loss of motivation, a lack of effort, and emotional indifference mimic bradykinesia, bradyphrenia, and masked facial expression of PD in the absence of apathy[60]. Similarly, flattened affect and passivity can manifest as monotonous and reduced spontaneous speech, which can be functions of hypophonia or cognitive dysfunction.

Establishing the diagnosis of apathy can be challenging for additional reasons. Apathy, which can represent a transient clinical state or an isolated symptom or syndrome, is defined inconsistently in the literature and lacks standardized or validated criteria for apathy. Marin's criteria of reduced goal-directed behavior and cognition and emotional concomitants of goal-directed behavior are the most widely used[69]. Controversy over the roles of an emotional dimension and cognitive deficits make it difficult to apply these criteria in PD. Discrete criteria for apathy are also lacking in the DSM-IV-TR.

Several rating scales, recently reviewed by a Movement Disorder Society task force, assist with evaluation[75]. Rating instruments are generally useful as screening tools to be used 
with a secondary diagnostic interview. The rater (patient, caregiver, or clinician) influences the quality of the information obtained. For example, patients with apathy may be indifferent as to whether they have undergone behavioral changes. Distinguishing apathy from depression requires evidence for emotional features such as low mood, a reduced sense of pleasure, guilt, diminished self-attitude, and anxiety in patients with a concurrent depressive disorder.

The diagnosis of fatigue is challenging because of its recognition as an independent entity that can also be a feature of other disturbances. For example, fatigue is one of the criteria for diagnosing major depression. It can be impossible to distinguish fatigue due to depression from that related to other factors. However, assessments of fatigue must account for the possibility of a depressive disturbance.

A number of fatigue rating scales have been developed for the general population and for specific conditions[76]. Unidimensional fatigue scales provide a single score that represents the range of symptoms and behaviors whereas multidimensional scales provide a profile of various qualitative and quantitative aspects of fatigue. The Parkinson's Fatigue Scale (PFS) was developed as a disease specific scale to overcome limitations of generic scales, which do not account for motor symptoms and to measure the single construct of physical fatigue[71]. Most fatigue scales use set cut-off scores to define presence or absence of clinically significant fatigue.

\section{Management}

Optimal management of apathy and fatigue involves vigilance for the symptoms of each, use of informants to obtain information to establish clinical diagnoses, and judicious use of assessment tools to screen for the presence of non-motor symptoms. Treatments for both include illness education to families and patients about depression, fatigue, apathy, and cognitive disturbances in PD, behavioral strategies to maximize executive functions, and use of medications to treat mood disorders and cognitive disturbances; improvements in comorbid conditions may be sufficient to relieve apathy and fatigue when they do occur.

Studies on the specific treatment of apathy in PD are limited. Management is often difficult since patients are indifferent to the need to attend to their own health and well-being and their inactivity can be misconstrued as "characterological laziness"[77]. Non-pharmacologic strategies involve providing an individualized daily schedule and structure with varied activities and group experiences that help to maintain a satisfactory activity level and enrichment. Campbell and Duffy underscore the role of the family as a therapeutic resource as patients benefit from a "goal-directed, reality-based, and paternalistic approach that essentially allows the caregiver to function as an 'auxiliary executive"'[77]. Possible medications include dopamine agonists, psychostimulants, modafanil, dopamine agonists, and testosterone[60,62,77]. Deep brain stimulation effects on apathy are inconsistent[62].

Few studies have investigated treatment of fatigue. In placebo-controlled trials, methylphenidate had a favorable effect on fatigue[78] and l-dopa improved physical fatigue[79]. Dopamine agonists have been helpful for some, but fatigue worsened in the placebo group compared to those who initiated levodopa therapy in early course untreated $\mathrm{PD}[80]$. Whereas modafanil, a wake-promoting drug, improved excessive daytime sleepiness but not fatigue in PD in a double-blind placebo-controlled trial[81], nocturnally administered sodium oxybate improved fatigue and excessive daytime sleepiness in PD[82]. 


\section{Hallucinations and Psychosis}

\section{Definitions}

Psychotic symptoms include visuo-perceptual symptoms such as visual hallucination (VH), illusions, passage and presence phenomena (See table 2 for definition of these phenomena). Hallucinations may also occur from other modalities, mainly auditory, but usually in combination with VH. In addition, thought disorders, typically delusions, may occur. Psychotic symptoms may be mild or severe, occur together or single, be accompanied by insight of the pathological nature of the symptom or nor, and may be accompanied by affective and behavioural disturbances and require psychiatric hospitalization.

\section{Epidemiology}

Psychotic symptoms are a frequent occurrence in PD. The frequencies vary according to the definition used. If mild forms are included, psychotic symptoms may affect up to $50 \%$ of patients.[27] Studies on psychosis have mostly focused on visual hallucinations, the most common type of psychotic symptom in PD. However, hallucinations can occur in all sensory domains and delusions of various types are also relatively common[83]. Relatively few longitudinal studies of the course of PD exist. However, psychotic symptoms, once present, tend to be persistent and progressive[84].

\section{Clinical presentation}

The typical VHs consist of persons, who may or may not be familiar, and less often animals or objects. They may be numerous, but often single or few in numbers. They are usually complex, and stereotype in a given patient. They appear suddenly, often move, and usually seem very real. They usually vanish suddenly, sometimes when the patient tries to ascertain their reality by approaching or touching them, or asking other people to confirm their presence. They often occur in dim light, often at night. Insight in the pathological nature of the phenomenon may or may not be present. Lack of insight is more common in subjects with cognitive impairment.

Capgras and similar misidentification phenomena may also occur. Typically, the patient thinks that his spouse is someone else and not his or her spouse, which may create challenging situations with affective and behavioural changes. Other forms of delusions are relatively rare, but are often persecutory, or focus on infidelity.

The neuropsychiatric symptoms in PD tend to cluster into distinct syndromes. In a recent study, five clusters were identified. The largest group showed symptoms of mild depression, followed by a group with hallucinations and mild other symptoms. One group had sleep disturbances exclusively, and another showed apathy, anxiety and depression. A small group showed a variety of severe symptoms, including psychosis and agitation[85].

\section{Assessment and diagnosis}

Recently, consensus criteria for PD associated psychosis was proposed after a NIHsponsored workshop [86]. However, psychotic symptoms are not always reported voluntarily, and the clinician should ask for these phenomena. Some patients may deny or refuse to report these symptoms, or, in dementia, may have forgotten, and thus a caregiver should be questioned as well. The frequency, intensity and impact of the symptoms should be noted, as well as the situation and circumstances in which they occur, the detailed phenomenology, and any other accompanying symptoms such as cognitive impairment, depression, anxiety, sleep disorders. There are several rating scales in use, although no generally accepted ones. Item 2 of the UPDRS subscale 1 can be used, and also the Neuropsychiatric Inventory. More detailed assessments can be made using specific scales 
such as Parkinson Psychosis Rating Scale. An MDS Task Force recently reviewed the different scales, listed their strengths and weaknesses and provided some guidelines regarding recommendations[87].

\section{Aetiology}

Psychotic symptoms develop from a complex interplay of extrinsic and intrinsic factors. It was previously considered that $\mathrm{VH}$ in PD were caused by the dopaminergic medications. However, it is now generally accepted that although dopaminergic drugs, in particular dopamine agonists, can contribute to the emergence of psychosis, other factors may be more important, such as dementia and visuospatial impairment, as well as general factors such as old age and more advanced disease stage. Brain changes found to be associated with an increased risk for psychotic symptoms in PD include cholinergic deficits and Lewy bodies in the temporal lobe. Pathological excitation of the visual pathways due to physical or chemical factors may contribute. Psychotic symptoms are frequent during night and associated with sleep-disturbances, and vivid dreams and sleep-related phenomena may lead to hallucinations[88].

\section{Clinical consequences}

The impact of psychosis is substantial in that it is associated with dementia, depression, earlier mortality, greater caregiver strain, and nursing home placement $[1,89]$. Thus, it is crucial to identify these symptoms in order to optimize the management of PD patients.

\section{Management}

Non-pharmacological-Psycho-educative approaches, such as information and guidance about the nature of the phenomena may help, as well as cognitive approaches such as distraction or re-directing attention. Environmental interventions such as improving lightconditions and visual aids may also be useful [90]. Searching for potential contributing medical factors is always important, such as pain, infection, dehydration, metabolic disturbance, sensory deficits, and recent changes in the medication.

Pharmacological-First line is always to attempt adjustment of the antiparkinson treatment. Reduction of dose or number of drugs may reduce the symptoms, even without worsening of motor symptoms. According to expert opinion, anticholinergics should be withdrawn first. It is usually recommended to withdraw selegiline, amantadine and dopamine agonists before changing the levo-dopa dosage.

The only adequately tested drug to recommend is clozapine, which has been shown to improve VH without worsening motor symptoms[91]. However, randomised placebocontrolled trials have been conducted in patients without dementia only. The risk of agranulocytosis necessitates regular blood test. Other antipsychotic agents such as risperidone and olanzapine, are less useful, due to being less effective and with a higher risk for adverse events including motor worsening, cognitive decline and, drowsiness and confusion[40]. Initial open-label reports on quetiapine were promising, although two placebo-controlled trials were negative. More novel antipsychotics, such as ziprasidone and aripiprazole, have not yet been tested systematically and preliminary reports are inconclusive. For a review, see [92]. Careful monitoring is required, and dosing should be low with small increments. Recent reports have high-lighted that antipsychotic agents have a risk of cerebrovascular incidents and increased mortality, as well as worsening of cognition, in elderly people with dementia[93].

Cholinergic agents may improve neuropsychiatric symptoms in PD, but there is yet no evidence-base to supporting their use for VH in PD. However, rivastigmine seems to be 
particularly useful for PD with $\mathrm{VH}$, in that the rapid cognitive decline in this group can be reduced[94].

\section{Acknowledgments}

The authors want to thank Ms Karen Simonsen for expert technical assistance.

Financial Disclosure: Dr. Marsh is supported by NIH grants [RO1-MH069666 and the Morris K. Udall Parkinson's Disease Research Center of Excellence at Johns Hopkins (NIH-P50-NS-58377)].

\section{References}

1. Aarsland D, Larsen JP, Lim NG, et al. Range of neuropsychiatric disturbances in patients with Parkinson's disease. J Neurol Neurosurg Psychiatry. 1999; 67:492-96. [PubMed: 10486397]

2. Schrag A, Jahanshahi M, Quinn NP. What contributes to depression in Parkinson's disease? PsycholMed. 2001; 31:65-73.

3. Reijnders JS, Ehrt U, Weber WE, Aarsland D, Leentjens AF. A systematic review of prevalence studies of depression in Parkinson's disease. Mov Disord. 2008; 23(2):183-89. [PubMed: 17987654]

4. Starkstein SE, Leentjens AFG. The nosological position of apathy in clinical practice. J Neurol Neurosurg Psychiatry. 2008; 79:1088-92. [PubMed: 18187477]

5. Weintraub D, Moberg PJ, Duda JE, Katz IR, Stern MB. Recognition and treatrment of depression in Parkinson's disease. JGeriatrPsychiatry Neurol. 2003; 16(3):178-83.

6. Menza MA, Robertson-Hoffman DE, Bonapace AS. Parkinson's disease and anxiety: comorbidity with depression. BiolPsychiatry. 1993; 34(7):465-70.

7. Richard IH. Anxiety disorders in Parkinson's disease. AdvNeurol. 2005; 96:42-55.

8. Leentjens AF, Dujardin K, Marsh L, et al. Anxiety rating scales in Parkinson's disease: Critique and recommendations. Mov Disord. 2008

9. Stein MB, Heuser IJ, Juncos JL, Uhde TW. Anxiety disorders in patients with Parkinson's disease. AmJ Psychiatry. 1990; 147(2):217-20. [PubMed: 2301664]

10. Nuti A, Ceravolo R, Piccinni A, et al. Psychiatric comorbidity in a population of Parkinson's disease patients. EurJ Neurol. 2004; 11(5):315-20. [PubMed: 15142224]

11. Lauterbach EC, Freeman A, Vogel RL. Differential DSM-III psychiatric disorder prevalence profiles in dystonia and Parkinson's disease. J Neuropsychiatry Clin Neurosci. 2004; 16(1):29-36. [PubMed: 14990756]

12. Witjas T, Kaphan E, Azulay JP, et al. Nonmotor fluctuations in Parkinson's disease: frequent and disabling. Neurology. Aug 13; 2002 59(3):408-13. [PubMed: 12177375]

13. Nissenbaum H, Quinn NP, Brown RG, Toone B, Gotham AM, Marsden CD. Mood swings associated with the 'on-off' phenomenon in Parkinson's disease. Psychol Med. Nov; 1987 17(4): 899-904. [PubMed: 3432464]

14. Siemers ER, Shekhar A, Quaid K, Dickson H. Anxiety and motor performance in Parkinson's disease. Mov Disord. Oct; 1993 8(4):501-6. [PubMed: 8232361]

15. Maricle RA, Nutt JG, Valentine RJ, Carter JH. Dose-response relationship of levodopa with mood and anxiety in fluctuating Parkinson's disease: a double-blind, placebo-controlled study. Neurology. Sep; 1995 45(9):1757-60. [PubMed: 7675241]

16. Ishihara L, Brayne C. A systematic review of depression and mental illness preceding Parkinson's disease. Acta Neurol Scand. 2006; 113(4):211-20. [PubMed: 16542159]

17. Braak H, Ghebremedhin E, Rub U, Bratzke H, Del Tredici K. Stages in the development of Parkinson's disease-related pathology. Cell Tissue Res. Oct; 2004 318(1):121-34. [PubMed: 15338272]

18. Paulus W, Jellinger K. The neuropathologic basis of different clinical subgroups of Parkinson's disease. J Neuropathol Exp Neurol. Nov; 1991 50(6):743-55. [PubMed: 1748881]

19. Mayeux R. The "serotonin hypothesis" for depression in Parkinson's disease. Adv Neurol. 1990; 53:163-6. [PubMed: 2239456] 
20. Torack RM, Morris JC. The association of ventral tegmental area histopathology with adult dementia. Arch Neurol. May; 1988 45(5):497-501. [PubMed: 3358699]

21. Remy P, Doder M, Lees A, Turjanski N, Brooks D. Depression in Parkinson's disease: loss of dopamine and noradrenaline innervation in the limbic system. Brain. Jun; 2005 128(Pt 6):131422. [PubMed: 15716302]

22. Arabia G, Grossardt BR, Geda YE, et al. Increased risk of depressive and anxiety disorders in relatives of patients with Parkinson disease. ArchGenPsychiatry. 2007; 64(12):1385-92.

23. Myslobodsky M, Lalonde FM, L HI. Are patients with Parkinson's disease suicidal? JGeriatrPsychiatry Neurol. 2001; 14:120-24.

24. Gotham AM, Brown RG, Marsden CD. Depression in Parkinson's disease: a quantitative and qualitative analysis. JNeurolNeurosurgPsychiatry. 1986; 49:381-89.

25. Myslobodsky M, Lalonde FM, Hicks L. Are patients with Parkinson's disease suicidal? J Geriatr Psychiatry Neurol. 2001; 14(3):120-4. Fall. [PubMed: 11563434]

26. Moriarty J. Suicide following deep brain stimulation for Parkinson's disease. J Neurol NeurosurgPsychiatry. 2008; 79(8):851.

27. Soulas T, Gurruchaga JM, Palfi S, Cesaro P, Nguyen JP, Fenelon G. Attempted and completed suicides after subthalamic nucleus stimulation for Parkinson's disease. J Neurol NeurosurgPsychiatry. 2008; 79(8):952-54.

28. Voon V, Krack P, Lang AE, et al. A multicentre study on suicide outcomes following subthalamic stimulation for Parkinson's disease. Brain. Oct; 2008 131(Pt 10):2720-8. [PubMed: 18941146]

29. Nazem S, Siderowf AD, Duda JE, et al. Suicidal and death ideation in Parkinson's disease. Mov Disord. Aug 15; 2008 23(11):1573-9. [PubMed: 18618660]

30. Mondolo F, Jahanshahi M, Grana A, Biasutti E, Cacciatori E, Di Benedetto P. Evaluation of anxiety in Parkinson's disease with some commonly used rating scales. Neurol Sci. 2007; 28(5): 270-75. [PubMed: 17972042]

31. American Psychiatric A. Diagnostic and statistical manual of mental disorders (DSM IV). American Psychiatric Association; Washington D.C.: 1994.

32. Marsh LF, McDonald Wm Fau - Cummings J, Cummings JF, Ravina B. Provisional diagnostic criteria for depression in Parkinson's disease: report of an NINDS/NIMH Work Group. Mov Disord. Feb; 2006 21(2):148-58. [PubMed: 16211591]

33. Starkstein SE, Merello M, Jorge R, et al. A validation study of depressive syndromes in Parkinson's disease. Mov Disord. 2008; 23(4):538-46. [PubMed: 18074376]

34. Schrag A, Barone P, Brown RG, et al. Depression rating scales in Parkinson's disease: critique and recommendations. Mov Disord. 2007; 22(8):1077-92. [PubMed: 17394234]

35. Cole K, Vaughan FL. The feasibililty of using cognitive behaviour therapy for depression associated with Parkinson's disease: a literature review. Parkinsonism and Related Disorders. 2005; 11:269-76. [PubMed: 15970452]

36. Frisina PG, Tenenbaum HR, Borod JC, Foldi NS. The effects of antidepressants in Parkinson's disease: a meta-analysis. Int J Neurosci. 2008; 118(5):667-82. [PubMed: 18446583]

37. Miyasaki JM, Martin W, Suchowersky O, Weiner WJ, Lang AE. Practice parameter: initiation of treatment for Parkinson's disease: an evidence-based review: report of the Quality Standards Subcommittee of the American Academy of Neurology. Neurology. 2002; 58(1):11-17. [PubMed: 11781398]

38. Weintraub D, Morales KH, Moberg PJ, et al. Antidepressant studies in Parkinson's disease: a review and meta-analysis. MovDisord. 2005 10.1002/mds.20555.

39. Pahwa R, Stacy MA, Factor SA, et al. Ropinirole 24-hour prolonged release: randomized, controlled study in advanced Parkinson disease. Neurology. Apr 3; 2007 68(14):1108-15. [PubMed: 17404192]

40. Miyasaki JM, Shannon K, Voon V, et al. Practice Parameter: evaluation and treatment of depression, psychosis, and dementia in Parkinson disease (an evidence-based review): report of the Quality Standards Subcommittee of the American Academy of Neurology. Neurology. 2006; 66(7):996-1002. [PubMed: 16606910]

41. Menza M, Defronzo Dobkin R, Marin H, et al. A controlled trial of antidepressants in patients with Parkinson disease and depression. Neurology. Dec 17.2008 
42. Devos D, Dujardin K, Poirot I, et al. Comparison of desipramine and citalopram treatments for depression in Parkinson's disease: a double-blind, randomized, placebo-controlled study. Mov Disord. Apr 30; 2008 23(6):850-7. [PubMed: 18311826]

43. Voon V, Lang AE. Antidepressants in the treatment of psychosis with comorbid depression in Parkinson disease. ClinNeuropharmacol. 2004; 27:90-92.

44. Straits-Troster K, Fields JA, Wilkinson SB, et al. Health-related quality of life in Parkinson's disease after pallidotomy and deep brain stimulation. Brain Cogn. 2000; 42(3):399-416. [PubMed: 10753487]

45. de Beurs E, Wilson KA, Chambless DL, Goldstein AJ, Feske U. Convergent and divergent validity of the Beck Anxiety Inventory for patients with panic disorder and agoraphobia. DepressAnxiety. 1997; 6(4):140-46.

46. da Silva TM, Munhoz RP, Alvarez C, et al. Depression in Parkinson's disease: a double-blind, randomized, placebo-controlled pilot study of omega-3 fatty-acid supplementation. J Affect Disord. Dec; 2008 111(2-3):351-9. [PubMed: 18485485]

47. Paus S, Schmitz-Hubsch T, Wullner U, Vogel A, Klockgether T, Abele M. Bright light therapy in Parkinson's disease: a pilot study. Mov Disord. Jul 30; 2007 22(10):1495-8. [PubMed: 17516492]

48. Cardoso EF, Fregni F, Martins Maia F, et al. rTMS treatment for depression in Parkinson's disease increases BOLD responses in the left prefrontal cortex. Int J Neuropsychopharmacol. Mar; 2008 11(2):173-83. [PubMed: 17708780]

49. Fregni F, Santos CM, Myczkowski ML, et al. Repetitive transcranial magnetic stimulation is as effective as fluoxetine in the treatment of depression in patients with Parkinson's disease. J Neurol Neurosurg Psychiatry. Aug; 2004 75(8):1171-4. [PubMed: 15258224]

50. Epstein CM, Evatt ML, Funk A, et al. An open study of repetitive transcranial magnetic stimulation in treatment-resistant depression with Parkinson's disease. Clin Neurophysiol. Oct; 2007 118(10):2189-94. [PubMed: 17714987]

51. Moellentine C, Rummans T, Ahlskog JE, et al. Effectiveness of ECT in patients with parkinsonism. J Neuropsychiatry Clin Neurosci. 1998; 10(2):187-93. Spring. [PubMed: 9608407]

52. van der Wurff FB, Stek ML, Hoogendijk WJ, Beekman AT. The efficacy and safety of ECT in depressed older adults: a literature review. Int J Geriatr Psychiatry. Oct; 2003 18(10):894-904. [PubMed: 14533122]

53. Ozer F, Meral H, Aydin B, Hanoglu L, Aydemir T, Oral T. Electroconvulsive therapy in druginduced psychiatric states and neuroleptic malignant syndrome. J Ect. Jun; 2005 21(2):125-7. [PubMed: 15905757]

54. Dujardin K, Sockeel P, Devos D, et al. Characteristics of apathy in Parkinson's disease. Mov Disord. 2007; 22(6):778-84. [PubMed: 17290451]

55. Levy ML, Cummings JL, Fairbanks LA, et al. Apathy is not depression. J Neuropsychiatry Clin Neurosci. 1998; 10(3):314-19. [PubMed: 9706539]

56. Starkstein SE, Mayberg HS, Preziosi TJ, Andrezejewski P, Leiguarda R, Robinson RG. Reliability, validity, and clinical correlates of apathy in Parkinson's disease. J Neuropsychiatry Clin Neurosci. 1992; 4(2):134-39. [PubMed: 1627973]

57. Kirsch-Darrow L, Fernandez HH, Marsiske M, Okun MS, Bowers D. Dissociating apathy and depression in Parkinson disease. Neurology. 2006; 67(1):33-38. [PubMed: 16832074]

58. Isella V, Melzi P, Grimaldi M, et al. Clinical, neuropsychological, and morphometric correlates of apathy in Parkinson's disease. Mov Disord. 2002; 17(2):366-71. [PubMed: 11921125]

59. Pluck GC, Brown RG. Apathy in Parkinson's disease. J Neurol Neurosurg Psychiatry. 2002; 73:636-42. [PubMed: 12438462]

60. Shulman LM. Apathy in patients with Parkinson's disease. International Review of Psychiatry. 2000; 12(4):298-306.

61. Friedman J, Friedman HS. Fatigue in Parkinson's disease. Neurology. 1993; 43:2016-18. [PubMed: 8413960]

62. Friedman JH, Brown RG, Comella C, et al. Fatigue in Parkinson's disease: a review. Mov Disord. 2007; 22(3):297-308. [PubMed: 17133511]

63. Herlofson K, Larsen JP. The influence of fatigue on health-related quality of life in patients with Parkinson's disease. Acta Neurol Scand. 2003; 107(1):1-6. [PubMed: 12542506] 
64. Alves G, Wentzel-Larsen T, Larsen JP. Is fatigue an independent and persistent symptom in patients with Parkinson disease? Neurology. 2004; 63(10):1908-11. [PubMed: 15557510]

65. Lou JS, Kearns G, Oken B, Sexton G, Nutt J. Exacerbated physical fatigue and mental fatigue in Parkinson's disease. Mov Disord. 2001; 16(2):190-96. [PubMed: 11295769]

66. Friedman JH, Chou KL. Sleep and fatigue in Parkinson's disease. Parkinsonism \& Related Disorders. 2004; 10(Suppl 1):S27-S35. [PubMed: 15109584]

67. Rochester L, Hetherington V, Jones D, et al. Attending to the task: interference effects of functional tasks on walking in Parkinson's disease and the roles of cognition, depression, fatigue, and balance. Arch PhysMedRehabil. 2004; 85(10):1578-85.

68. Shulman LM, Taback RL, Rabinstein AA, Weiner WJ. Non-recognition of depression and other non-motor symptoms in Parkinson's disease. Parkinsonism and Related Disorders. 2002; 8:19397. [PubMed: 12039431]

69. Marin RS. Differential diagnosis of apathy and related disorders of diminished motivation. Psychiatric Annals. 1997; 27(1):30-33.

70. Marin RS. Differential diagnosis and classification of apathy. AmericanJournal of Psychiatry. 1990; 147(1):22-30. [PubMed: 2403472]

71. Brown RG, Dittner A, Findley L, Wessely SC. The Parkinson fatigue scale. Parkinsonism Relat Disord. 2005; 11(1):49-55. [PubMed: 15619463]

72. Martinez-Martin P, Catalan MJ, Benito-Leon J, et al. Impact of fatigue in Parkinson's disease: the Fatigue Impact Scale for Daily Use (D-FIS). QualLife Res. 2006; 15(4):597-606.

73. Zesiewicz TA, Patel-Larson A, Hauser RA, Sullivan KL. Social Security Disability Insurance (SSDI) in Parkinson's disease. DisabilRehabil. 2007; 29(24):1934-36.

74. Karlsen K, Larsen JP, Tandberg E, Jorgensen K. Fatigue in patients with Parkinson's disease. Mov Disord. 1999; 14(2):237-41. [PubMed: 10091615]

75. Leentjens AFG, Dujardin K, Marsh L, et al. Apathy and anhedonia rating scales in Parkinson's disease: critique and recommendations. Mov Disord. 2008; 23(14):2004-14. [PubMed: 18709683]

76. Dittner AJ, Wessely SC, Brown RG. The assessment of fatigue: a practical guide for clinicians and researchers. J PsychosomRes. 2004; 56(2):157-70.

77. Campbell JJ, Duffy JD. Treatment strategies in amotivated patients. Psychiatric Annals. 1997; 27(1):44-49.

78. Mendonca DA, Menezes K, Jog MS. Methylphenidate improves fatigue scores in Parkinson disease: a randomized controlled trial. Mov Disord. 2007; 22(14):2070-76. [PubMed: 17674415]

79. Lou JS, Kearns G, Benice T, Oken B, Sexton G, Nutt J. Levodopa improves physical fatigue in Parkinson's disease: a double-blind, placebo-controlled, crossover study. Mov Disord. 2003; 18(10):1108-14. [PubMed: 14534913]

80. Schifitto G, Friedman JH, Oakes D, et al. Fatigue in levodopa-naive subjects with Parkinson disease. Neurology. 2008; 71:481-85. [PubMed: 18695158]

81. Adler CH, Caviness JN, Hentz JG, Lind M, Tiede J. Randomized trial of modafinil for treating subjective daytime sleepiness in patients with Parkinson's disease. Mov Disord. 2003; 18(3):28793. [PubMed: 12621632]

82. Ondo WG, Perkins T, Swick T, et al. Sodium oxybate for excessive daytime sleepiness in Parkinson's disease. An open-label polysomnographic study. Arch Neurol. 2008; 65(10):1337-40. [PubMed: 18852348]

83. Graham JM, Grunewald RA, Sagar HJ. Hallucinosis in idiopathic Parkinson's disease. Journal of neurology, neurosurgery, and psychiatry. Oct; 1997 63(4):434-40.

84. Factor SA, Feustel PJ, Friedman JH, et al. Longitudinal outcome of Parkinson's disease patients with psychosis. Neurology. Jun 10; 2003 60(11):1756-61. [PubMed: 12796526]

85. Bronnick K, Aarsland D, Larsen JP. Neuropsychiatric disturbances in Parkinson's disease clusters in five groups with different prevalence of dementia. Acta psychiatrica Scandinavica. Sep; 2005 112(3):201-7. [PubMed: 16095475]

86. Ravina B, Marder K, Fernandez HH, et al. Diagnostic criteria for psychosis in Parkinson's disease: report of an NINDS, NIMH work group. Mov Disord. Jun 15; 2007 22(8):1061-8. [PubMed: 17266092] 
87. Fernandez HH, Aarsland D, Fenelon G, et al. Scales to assess psychosis in Parkinson's disease: Critique and recommendations. Mov Disord. Mar 15; 2008 23(4):484-500. [PubMed: 18175343]

88. Onofrj M, Thomas A, Bonanni L. New approaches to understanding hallucinations in Parkinson's disease: phenomenology and possible origins. Expert review of neurotherapeutics. Dec; 2007 7(12):1731-50. [PubMed: 18052766]

89. Aarsland D, Larsen JP, Tandberg E, Laake K. Predictors of nursing home placement in Parkinson's disease: a population-based, prospective study. Journal of the American Geriatrics Society. Aug; 2000 48(8):938-42. [PubMed: 10968298]

90. Diederich NJ, Pieri V, Goetz CG. Coping strategies for visual hallucinations in Parkinson's disease. Mov Disord. Jul; 2003 18(7):831-2. [PubMed: 12815665]

91. Low-dose clozapine for the treatment of drug-induced psychosis in Parkinson's disease. The Parkinson Study Group. The New England journal of medicine. Mar 11; 1999 340(10):757-63. [PubMed: 10072410]

92. Zahodne LB, Fernandez HH. Course, prognosis, and management of psychosis in Parkinson's disease: are current treatments really effective? CNS spectrums. Mar; 2008 13(3 Suppl 4):26-33. [PubMed: 18323764]

93. Ballard C, Waite J. The effectiveness of atypical antipsychotics for the treatment of aggression and psychosis in Alzheimer's disease. Cochrane database of systematic reviews (Online). 2006; (1):CD003476. [PubMed: 16437455]

94. Burn D, Emre M, McKeith I, et al. Effects of rivastigmine in patients with and without visual hallucinations in dementia associated with Parkinson's disease. Mov Disord. Nov; 2006 21(11): 1899-907. [PubMed: 16960863] 


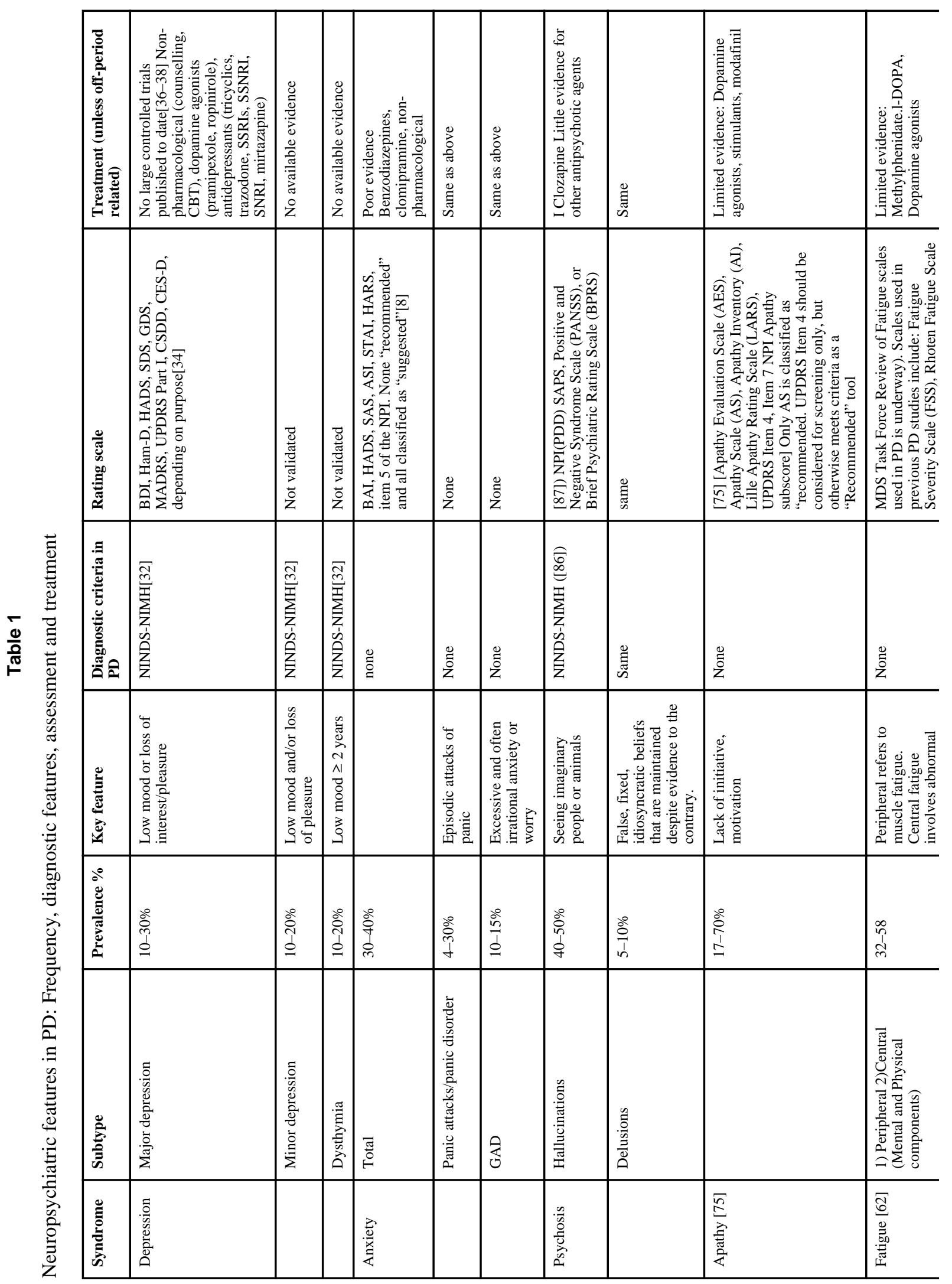




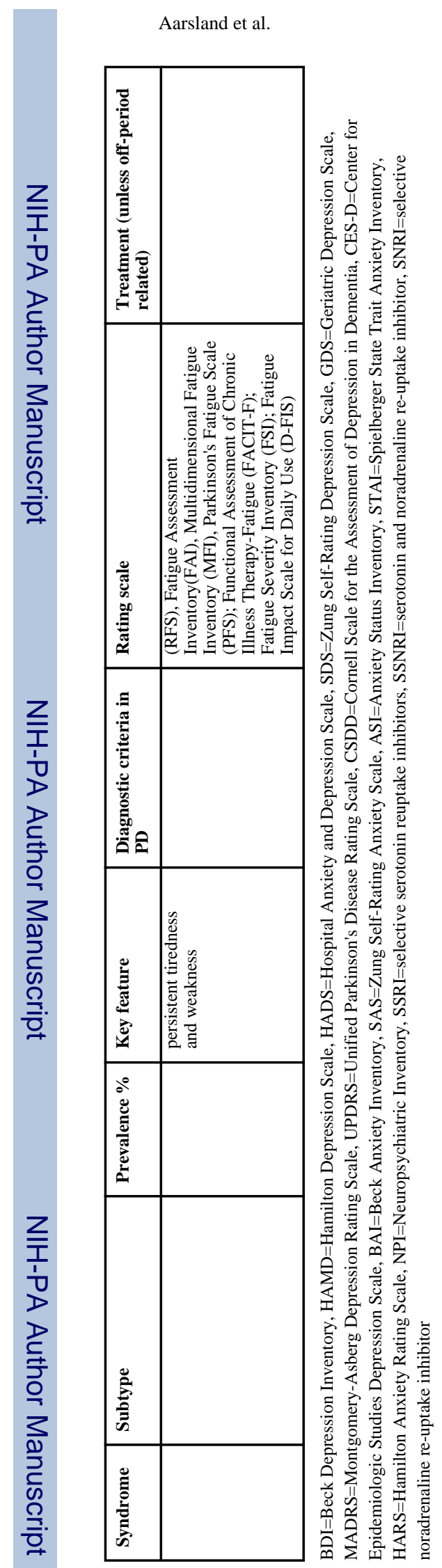

Mov Disord. Author manuscript; available in PMC 2010 November 15. 
Table 2

Terms and definitions used in this critique (from ref [87]):

\begin{tabular}{|l|l|}
\hline Term & Definition \\
\hline Psychosis & $\begin{array}{l}\text { the full spectrum of features seen in PD psychosis, including hallucinations and delusions and the "minor" } \\
\text { phenomena of illusions, 'passage hallucinations' and 'sense of presence' }\end{array}$ \\
\hline Hallucinations: & $\begin{array}{l}\text { are abnormal perceptions without a physical stimulus that can involve any sensory modality and may be simple or } \\
\text { complex in form. }\end{array}$ \\
\hline Illusions & are misperceptions of real stimuli that are often visual in nature \\
\hline Delusions: & false, fixed, idiosyncratic beliefs that are maintained despite evidence to the contrary. \\
\hline Presence hallucinations & consist of the experience that someone is present when nobody is actually there \\
\hline Passage hallucinations & fleeting, vague imaging in the peripheral vision \\
\hline
\end{tabular}

